\title{
Throughput Benefit Assessment for Tactical Runway Configuration Management (TRCM)
}

\author{
Nipa Phojanamongkolkij ${ }^{1}$, Rosa M. Oseguera-Lohr ${ }^{2}$, Gary W. Lohr ${ }^{3}$ \\ NASA Langley Research Center, Hampton, VA, 23681,USA \\ and \\ James W. Fenbert ${ }^{4}$ \\ Analytical Mechanics Associates, Inc.,Hampton,VA, 23681, USA
}

\begin{abstract}
The System-Oriented Runway Management (SORM) concept is a collection of needed capabilities focused on a more efficient use of runways while considering all of the factors that affect runway use. Tactical Runway Configuration Management (TRCM), one of the SORM capabilities, provides runway configuration and runway usage recommendations, monitoring the active runway configuration for suitability given existing factors, based on a 90 minute planning horizon. This study evaluates the throughput benefits using a representative sample of today's traffic volumes at three airports: Memphis International Airport (MEM), Dallas-Fort Worth International Airport (DFW), and John F. Kennedy International Airport (JFK). Based on this initial assessment, there are statistical throughput benefits for both arrivals and departures at MEM with an average of $4 \%$ for arrivals, and $6 \%$ for departures. For DFW, there is a statistical benefit for arrivals with an average of $3 \%$. Although there is an average of $1 \%$ benefit observed for departures, it is not statistically significant. For JFK, there is a $12 \%$ benefit for arrivals, but a $2 \%$ penalty for departures. The results obtained are for current traffic volumes and should show greater benefit for increased future demand. This paper also proposes some potential TRCM algorithm improvements for future research. A continued research plan is being worked to implement these improvements and to re-assess the throughput benefit for today and future projected traffic volumes.
\end{abstract}

\section{Nomenclature}

$\begin{array}{ll}\text { AAR } & =\text { Airport Acceptance Rate } \\ \text { ADR } & =\text { Airport Departure Rate } \\ \text { ANCOVA } & \text { Analysis of Covariance } \\ \text { ASPM } & =\text { Aviation System Performance Metrics } \\ \text { CADRS } & =\text { Combined Arrival/Departure Runway Scheduling } \\ \text { DFW } & =\text { Dallas-Fort Worth International Airport } \\ \text { FAA } & =\text { Federal Aviation Administration } \\ \text { IMC } & =\text { Instrument Meteorological Condition } \\ \text { JFK } & =\text { John F. Kennedy International Airport } \\ \text { JPDO } & =\text { Joint Planning and Development Office } \\ \text { MEM } & =\text { Memphis International Airport } \\ \text { MSE } & =\text { Metroplex Simulation Environment }\end{array}$

${ }^{1}$ Operations Research Analyst, Systems Engineering and Engineering Methods Branch, NASA Langley Research Center, M.S. 442, Hampton, VA.

${ }^{2}$ Aerospace Engineer, Aeronautics Systems Analysis Branch, NASA Langley Research Center, M.S. 442, Hampton, VA 23681, Senior Member AIAA.

${ }^{3}$ Senior Aerospace Research Engineer, Crew Systems and Aviation Operations Branch, NASA Langley Research Center, M.S. 152, Hampton, VA 23681, Senior Member AIAA.

${ }^{4}$ Aerospace Engineer, Analytical Mechanics Associates, Inc.,Hampton,VA, 23681, USA. 
NAS $=$ National Airspace System

NextGen $=$ Next Generation Air Transportation System

RCM = Runway Configuration Management

SORM = System-Oriented Runway Management

SRCM = Strategic Runway Configuration Management

$\mathrm{TE}=$ Throughput Efficiency

TRCM = Tactical Runway Configuration Management

VMC $=$ Visual Meteorological Condition

\section{Introduction}

$\mathrm{D}$ EMAND for air transportation and other airspace services is expected to grow significantly from today's levels in terms of passenger volume, amount of cargo shipped, and overall number of flights. To support this projected growth, goals for the Next Generation Air Transportation System (NextGen) are to significantly increase the safety, security, capacity, efficiency, and environmental compatibility of air transportation operations [1]. These benefits can be achieved through a combination of new procedures and advances in the technology deployed to manage passenger, air cargo, and air traffic operations. New concepts, leveraging on new technologies, focused on increasing safety and efficiency in the National Airspace System (NAS), are under investigation. These concepts include those designed to increase capacity for operations in enroute airspace as well as in terminal airspace (including departure and arrival operations). As concepts focused on efficiency of operations in various air traffic domains progress, efficient use of runways becomes paramount. This means selecting runway configurations that contribute to systemic (NAS) efficiency and local airport needs, as well as optimizing operations across selected runway configurations (i.e. how runways are used, for departures, arrivals, or mixed).

The System-Oriented Runway Management (SORM) concept is a collection of needed capabilities focused on ensuring efficient use of runways while considering all of the factors that affect runway use. SORM is composed of two basic capabilities: Runway Configuration Management (RCM), and Combined Arrival/Departure Runway Scheduling (CADRS). RCM is the process of designating active runways, monitoring the active runway configuration for suitability given existing factors, and predicting future configuration changes; CADRS is the process of distributing arrivals and departures across active runways based on local airport and NAS goals.

SORM further decomposes the RCM capabilities into Strategic RCM (SRCM) and Tactical RCM (TRCM). These capabilities correspond to the time scale in which they would operate: SRCM with a planning horizon on the order of several hours (six hours has been identified as a starting point for the investigation of SORM capabilities), TRCM with a planning horizon on the order of 90 minutes, and CADRS with a planning horizon on the order of 1530 minutes [2, 3].

Algorithm development was initiated in all three capabilities, but the most fully-developed to date is the TRCM algorithm. Initial evaluation of this algorithm was conducted by the developer (Mosaic ATM, Contract \# NNL09AA02B), using eight days of traffic data from late 2008 to 2009 at two airports, Hartsfield-Jackson Atlanta International Airport (ATL) and John F. Kennedy International Airport (JFK). Although results were not uniform across all days of traffic and weather, quantifiable benefits on transit time and delay to using TRCM at these two airports were documented.

This paper focuses on an initial assessment on throughput benefit using the current TRCM algorithm at today's traffic volume. Any insight on potential algorithm improvement from this assessment will be planned for the followup research. The organization of the paper is as follow. Section 2 gives a description of SORM and the TRCM algorithm. Following this description of the algorithm, method of test is presented (Section 3). Section 4 provides the results of this initial assessment. Conclusions and proposed future research are detailed in Section 5.

\section{Description of SORM and the TRCM Algorithm}

The fundamental elements of SORM are summarized here. Figure 1 gives an overview of how the SORM software operates. The current software requires three types of inputs, which are weather information, airport information, and flight plans. Weather information includes wind speed and wind direction. Airport information includes layout of terminals, spots ${ }^{5}$, runways, arrival fixes, departure fixes, and their associated latitude and longitude coordinates, available runway configurations, and the current active runway configuration schedule. Flight

\footnotetext{
${ }^{5}$ Spot is the position where hand-off responsibility transfers between non-movement area (or ramp) and movement area controllers.
} 
plan includes flight ID, origin and destination airports, assigned fix, scheduled arrival time at fix, and scheduled departure time at gate.

Figure 1 gives an overview of how the SORM software operates. The SORM Manager alternates the control between planning and execution cycles. During the planning cycle (the Planning in TRCM); it uses the TRCM algorithm with a low fidelity fast-time simulation to plan for the next 90 minutes. In the current algorithm, runway configuration change is not allowed during the freezing interval (the first 45-minute interval). The TRCM algorithm produces a plan listing recommended runway configurations and their associated change schedules during the planning interval (the last 45-minute interval). If the algorithm cannot find a better configuration than the current configuration, then the plan will be an empty list.

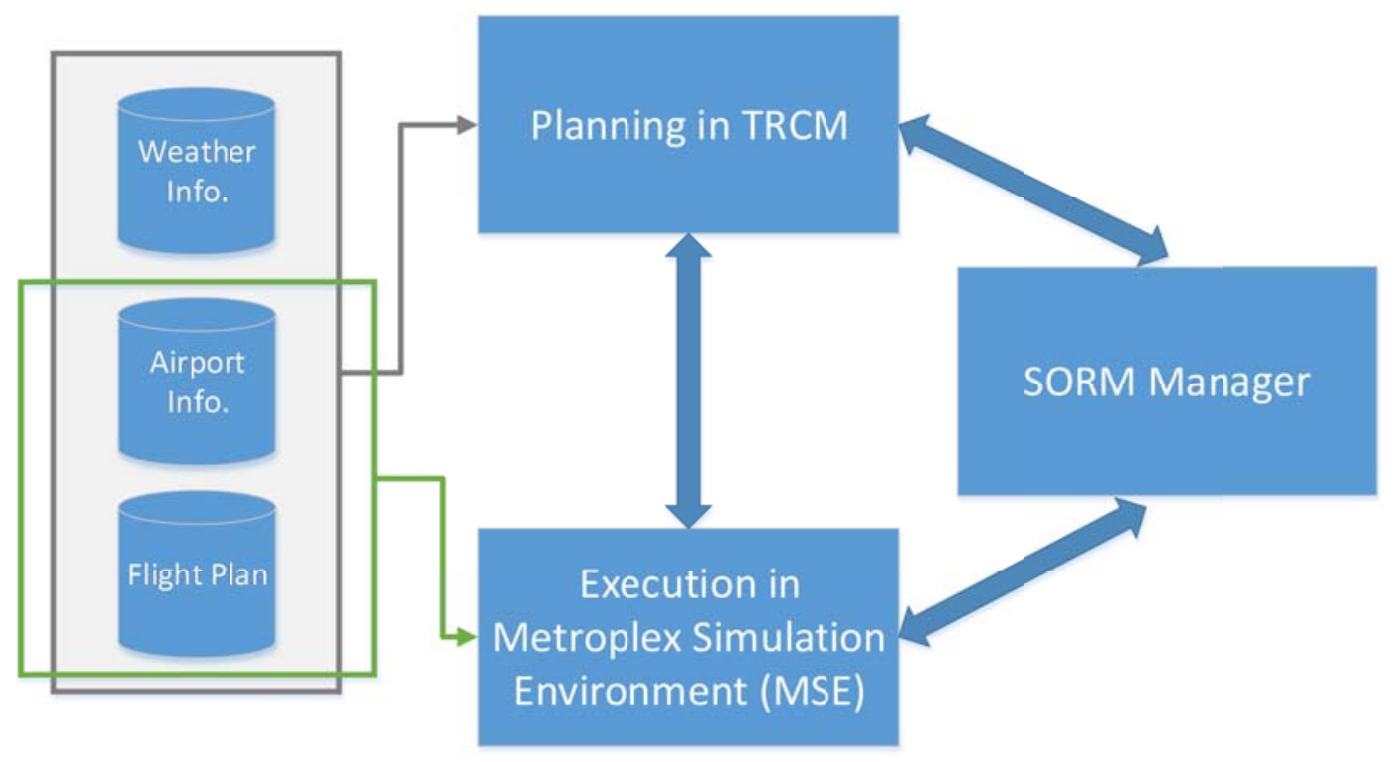

Figure 1. Overview of the current SORM software.

A basic concept of the TRCM algorithm uses an enumerative search of all available runway configurations to find the optimum configuration that minimizes the total transit time ${ }^{6}$ across all flights. During the enumerative search of the algorithm, the low fidelity simulation environment is used to calculate the total transit time (the objective value). The algorithm trades off the airborne, taxi, and queue (departing aircraft only) time durations in search for the minimum total time. The low fidelity simulation computes transit time durations using the great circle distances for all airborne and taxi routes, along with their corresponding speeds. Wind speed and direction are taken into account to penalize runway configurations that should not be used during unfavorable wind conditions. This is accomplished by adding a penalty to the total transit time. The algorithm output provides: the optimum (minimum total transit time) runway configuration; the time at which the optimum configuration should be used; the first flight on the optimum configuration; and the last flight on the current configuration. The current algorithm, however, recommends a change from the current to the optimum configuration even if there is only a total transit time saving of as low as 1 second, or there is as few as one aircraft benefit from the change. This can easily be improved by setting some minimal threshold for recommending a configuration change. Other limitations are: the simulation includes no runway crossing time delay; and uses a predefined constant airborne speed and constant taxi speed for all flights.

After each iteration of the planning cycle, the SORM software then operates in the execution cycle (the Execution in MSE) simulating the airborne and taxi routes for individual aircraft entirely using a high fidelity simulation environment, called the Metroplex Simulation Environment (MSE). It uses the airport information and flight plan along with the runway configuration determined in the most recent planning cycle as inputs to simulate the arrival and departure operations. The current high fidelity MSE includes high-level of detail for airport surface

\footnotetext{
${ }^{6}$ Transit time is defined as the travel time from arrival fix to spot (for arriving aircraft), or from spot to departure fix (for departing aircraft).
} 
operations using node-link ${ }^{7}$ connections replicating the actual airport surface layout. It also incorporates aircraftspecific airborne-speed and taxi-speed profiles. The MSE records several flight's status, including all simulated flight routes and their time stamps at all predetermined surface nodes. It provides the latest flight's status to the planning cycle for its next iteration. These alternating cycles continue until all flights under study were simulated. Any performance metrics can be post-processed and computed from the MSE flight's records. The current MSE does not take any wind information into account during the simulation of arrival and departure operations. Table 1 compares features and scopes of the low fidelity fast-time simulation and the high fidelity MSE used in the SORM software.

Table 1. Simulation environment comparison.

\begin{tabular}{|l|l|l|}
\hline \multicolumn{1}{|c|}{ Features } & \multicolumn{1}{|c|}{ Low fidelity fast-time simulation } & \multicolumn{1}{c|}{ High fidelity MSE } \\
\hline $\begin{array}{l}\text { Usage phase of the } \\
\text { software }\end{array}$ & $\begin{array}{l}\text { Determine the optimum runway schedule } \\
\text { (planning). }\end{array}$ & $\begin{array}{l}\text { Execute the recommended optimum runway } \\
\text { schedule (executing) and records all flight's } \\
\text { status for the next planning iteration, and for } \\
\text { metric post-processing. }\end{array}$ \\
\hline Assumptions & $\begin{array}{l}\text { 1. Weather (wind) information is used to } \\
\text { penalize configurations that are not operable } \\
\text { under the certain weather conditions. }\end{array}$ & $\begin{array}{l}\text { 1. No weather (wind) information is used } \\
\text { during the simulation of the arrival and } \\
\text { departure operations. }\end{array}$ \\
\cline { 2 - 3 } 2. No runway crossing constraint. & $\begin{array}{l}\text { 2. Actual runway crossing constraints are } \\
\text { simulated. }\end{array}$ \\
\cline { 2 - 3 } & $\begin{array}{l}\text { 3. A predefined constant for taxi speed for all } \\
\text { aircraft. }\end{array}$ & 3. Aircraft-specific taxi-speed profiles. \\
\cline { 2 - 3 } & $\begin{array}{l}\text { 4. A predefined constant for airborne speed } \\
\text { for all aircraft. }\end{array}$ & 4. Aircraft-specific airborne-speed profiles. \\
\hline
\end{tabular}

\section{Method of Test}

\section{A. Test Cases}

For this assessment, a set of historical data that covers a period from late 2009 to 2010 was used. The selected days for testing are based on a set of twelve days identified by the Federal Aviation Administration (FAA) and the Joint Planning and Development Office (JPDO) for NextGen research. These days were identified by the FAA as representative traffics capturing seasonal trends in the NAS for that year, using an optimization based method to minimize differences in predicted and actual levels of desired performance metrics at the daily level [4]. Three airports-MEM, DFW, and JFK - were selected for this study. MEM was chosen because FAA planned to perform a field evaluation of the TRCM algorithm at MEM, and any initial benefit assessment at this airport would be valuable prior to the field test. JFK was selected largely due to its complex operations, and its location and dependency with respect to the other airports nearby. DFW was considered to be very different from the other two airports due to the larger traffic volume and fewer runway configuration options than JFK. In addition, the three airports have been identified (along with another 27 airports) as core airports for FAA's implementation of key NextGen operational improvement areas through 2018 [5].

Figure 2 shows airport diagrams of these airports. MEM has three parallel runways in the north/south orientation (36L/18R, 36C/18C, and 36R/18L) and one runway (9/27) on the north side of the airport. About 40\% of MEM traffic volumes are from FedEx airline. DFW has five parallel runways in the north/south orientation (36L/18R, 36R/18L, 35L/17R, 35C/17C, and 35R/17L), and two parallel runways in the southeast/northwest orientation (13R/31L and 13L/31R). About 80\% of DFW traffic volumes are from American Airline and American Eagle. JFK has two sets of parallel runways. One is in the southeast/northwest orientation $(13 \mathrm{R} / 31 \mathrm{~L}$ and $13 \mathrm{~L} / 31 \mathrm{R})$. The other is in the northeast/southwest orientation (4L/22R and $4 \mathrm{R} / 22 \mathrm{~L}$ ). Runway configurations evaluated in this study were based on the most frequent uses from the historical data. Table 2 gives a complete list of all evaluated configurations in this study. Runway configuration numbers 1 and 2 at MEM are A_18L_18R_D_18R_18C (arrivals on 18L and 18R, departures on 18R and 18C), and A_18L_(18R)_D_18R_18C (arrivals primary on 18L with an overflow traffic on 18R, departures on 18R and 18C). These two configurations present the dedicated uses of 18L for arrivals and

\footnotetext{
${ }^{7}$ Node-link connections represent an interconnected small surface segments that an aircraft can travel along. Stopping and holding are allowed at nodes. Continuous movement is only allowed on links.
} 
18C for departures, and the mixed use of 18R. There are four runway configurations evaluated at MEM, 10 configurations at DFW, and 20 configurations at JFK.
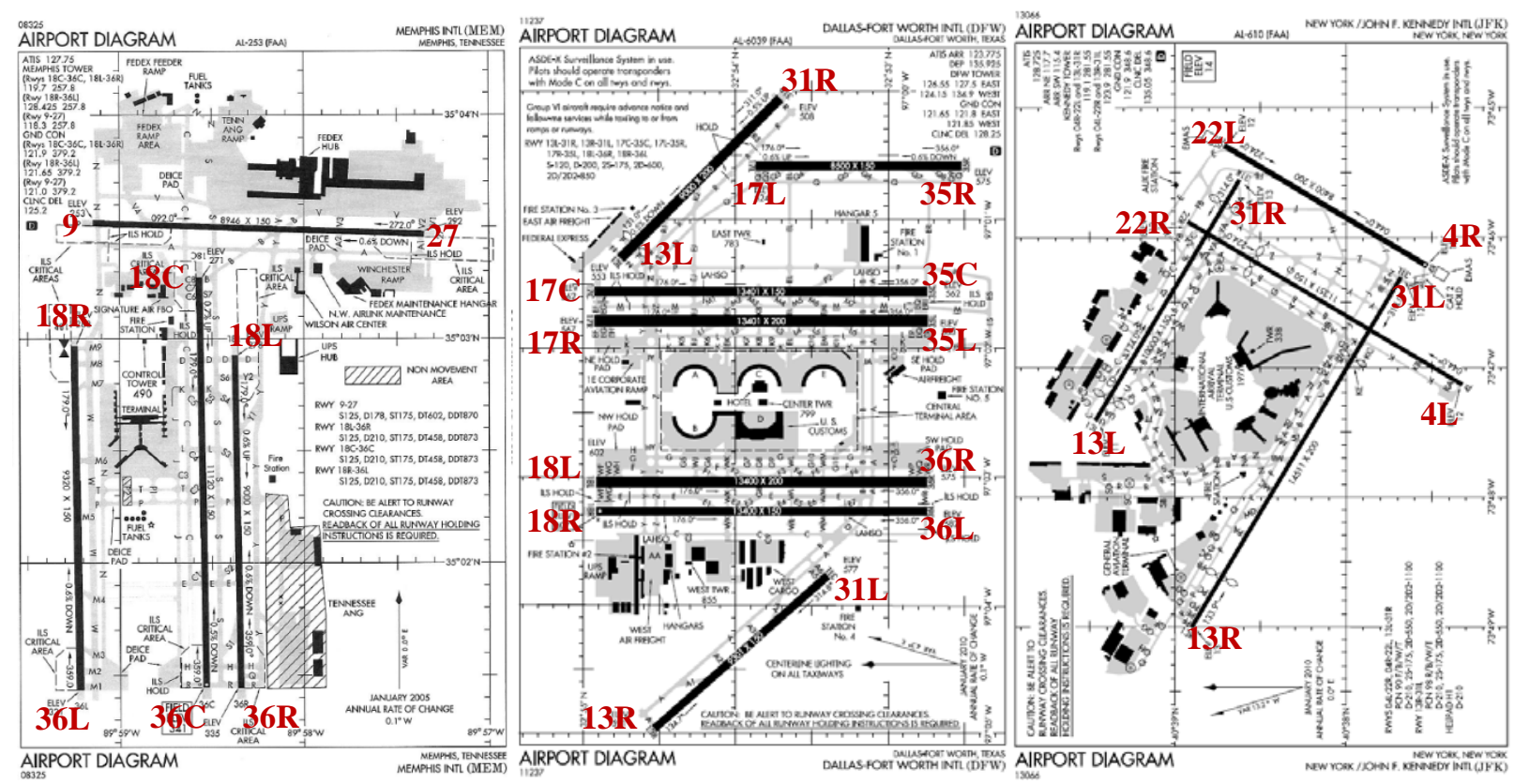

Figure 2. Airport Diagrams for MEM, DFW, and JFK, respectively, image courtesy of FAA.

Table 2. Runway Configurations.

(A_rwy1_(rwy2)_D_rwy3 = Arrivals primary on runway 1 with (overflow) secondary on runway 2; Departures on runway 3)

\begin{tabular}{|c|l|l|l|}
\hline $\begin{array}{c}\text { Configuration } \\
\text { Number }\end{array}$ & \multicolumn{1}{|c|}{ MEM } & \multicolumn{1}{|c|}{ DFW } & \multicolumn{1}{|c|}{ JFK } \\
\hline 1 & A_18L_18R_D_18R_18C & A_18R_17C_17L_13R_D_18L_17R & A_31R_31L_D_31L \\
\hline 2 & A_18L_(18R)_D_18R_18C & A_18R_17C_17L_D_18L_17R & A_13L_22L_D_13R \\
\hline 3 & A_36R_36L_D_36L_36C & A_18R_17C_13R_D_18L_17R & A_31R_D_31L \\
\hline 4 & A_36R_(36L)_D_36L_36C & A_18R_17C_D_18L_17R & A_22L_D_22R \\
\hline 5 & & A_36L_35C_35R_31R_D_36R_35L & A_13L_D_13R \\
\hline 6 & & A_36L_35C_35R_D_36R_35L & A_4R_D_4L \\
\hline 7 & & A_36L_35C_D_36R_35L & A_22L_22R_D_22R \\
\hline 8 & & A_36L_35C_35R_31R_D_36R_35L_31L & A_4R_4L_D_4L \\
\hline 9 & & A_36L_35C_35R_D_36R_35L_31L & A_13L_D_13R_13L \\
\hline 10 & & A_36L_35C_D_36R_35L_31L & A_4R_13R_D_4L \\
\hline 11 & & & A_4R_13R_D_13L \\
\hline 12 & & & A_13L_13R_D_13R_13L \\
\hline 13 & & & A_4R_D_13L \\
\hline 14 & & & A_22L_D_13R \\
\hline 15 & & & A_22L_D_22R_31L \\
\hline 16 & & & A_4R_D_4L_31L \\
\hline 17 & & & A_4R_4L_D_4L_31L \\
\hline 18 & & & A_22L_22R_D_22R_31L \\
\hline 19 & & & A_22L_22R_D_31L \\
\hline 20 & & & A_13L_D_22R \\
\hline
\end{tabular}


Table 3 provides detail of the twelve tested days. These days are representative of the 2009-2010 time period and cover a range of weather conditions and traffic volumes. The traffic volumes did not include military and general aviation, and therefore are lower than those reported in the ASPM database. There were 4 weekend days. MEM and JFK had approximately the same magnitude of traffic ( $\sim 500$ arrivals and 500 departures per day) for weekdays. DFW had approximately twice the volume as the other two airports. Weekend and weekday volumes did not significantly differ at JFK. However, the weekend volumes at MEM differed significantly from the weekday volumes. This is due to there being much fewer FedEx flights on weekends.

Table 3. Test Cases(A = Arrival Operations, $\mathbf{D}=$ Departure Operations, $\mathbf{A}+\mathbf{D}=$ Total Operations).

\begin{tabular}{|c|c|c|c|c|c|c|c|c|c|c|}
\hline Test Case & Date & \multicolumn{3}{|c|}{ MEM } & \multicolumn{3}{|c|}{ DFW } & \multicolumn{3}{c|}{ JFK } \\
\cline { 3 - 11 } Number & (day of the week) & \#A & \#D & A+D & \#A & \#D & A+D & \#A & \#D & A+D \\
\hline 1 & $10 / 06 / 2009(\mathrm{~T})$ & 511 & 471 & 982 & 804 & 789 & 1,593 & 425 & 420 & 845 \\
\hline 2 & $10 / 17 / 2009(\mathrm{SA})$ & 317 & 304 & 621 & 763 & 762 & 1,525 & 448 & 471 & 919 \\
\hline 3 & $11 / 20 / 2009(\mathrm{~F})$ & 539 & 458 & 997 & 892 & 887 & 1,779 & 522 & 517 & 1,039 \\
\hline 4 & $01 / 10 / 2010(\mathrm{~S})$ & 311 & 343 & 654 & 853 & 855 & 1,708 & 504 & 426 & 930 \\
\hline 5 & $03 / 09 / 2010(\mathrm{~T})$ & 541 & 465 & 1,006 & 879 & 893 & 1,772 & 499 & 478 & 977 \\
\hline 6 & $03 / 25 / 2010(\mathrm{TH})$ & 557 & 493 & 1,050 & 886 & 892 & 1,778 & 524 & 508 & 1,032 \\
\hline 7 & $05 / 06 / 2010(\mathrm{TH})$ & 556 & 494 & 1,050 & 904 & 909 & 1,813 & 490 & 478 & 968 \\
\hline 8 & $05 / 18 / 2010(\mathrm{~T})$ & 549 & 495 & 1,044 & 901 & 909 & 1,810 & 482 & 468 & 950 \\
\hline 9 & $06 / 05 / 2010(\mathrm{SA})$ & 330 & 315 & 645 & 792 & 786 & 1,578 & 478 & 482 & 960 \\
\hline 10 & $07 / 03 / 2010(\mathrm{SA})$ & 353 & 343 & 696 & 799 & 785 & 1,584 & 525 & 521 & 1,046 \\
\hline 11 & $07 / 13 / 2010(\mathrm{~T})$ & 561 & 498 & 1,059 & 908 & 911 & 1,819 & 500 & 475 & 975 \\
\hline 12 & $07 / 22 / 2010(\mathrm{TH})$ & 560 & 507 & 1,067 & 925 & 929 & 1,854 & 560 & 533 & 1,093 \\
\hline
\end{tabular}

It should be emphasized that these tested volumes do not represent future demand growth where the volume exceeds the current capacity. This paper is an initial study to determine if benefit can be realized at today's volumes. If there is a minimal benefit of using the TRCM at today's volume, potential, and perhaps airport-specific, algorithm improvement from this study will be planned for the future research. The revised TRCM algorithm will then be used to assess any benefit at today's and future projected volumes.

\section{B. Metric Definition}

The primary metric for this study is throughput efficiency. Throughput efficiency (TE) is the ratio of the actual to the optimum effective throughput, and is unit less. The actual effective throughput is defined as the ratio of the number of aircraft that have transited through its exit system point to the total actual travel time of these aircraft. For the arrival operation, the total travel time is the sum (over all arriving aircraft) of the difference between the actual time stamp ${ }^{8}$ at spot (where aircraft exit the system) and the earliest time stamp ${ }^{9}$ at arrival fix (where aircraft enter the system). For the departure operation, the total travel time is the sum (over all departing aircraft) of the difference between the actual time stamp at departure fix (where aircraft exit the system) and the actual time stamp at spot (where aircraft enter the system). The optimum effective throughput is defined similarly, with the exception of using the total unimpeded ${ }^{10}$ times instead of the total actual times. Due to availability of unimpeded times data from the MSE, the total travel time in this study only consists of the taxi times and the airborne times for the TE calculation. It is noticed that taxi and airborne times are the major drivers to the total travel time. Any system with TE closer to 1 is preferred. The TE can be thought of as a throughput metric that has been standardized so that it is insensitive to traffic volume [6].

\section{Data Collection}

To assess the TRCM benefit, the throughput metric defined in Section B must be compared with the actual historical operations. Because of the lower traffic volumes than those in the ASPM, the metrics of interest were not available from the ASPM for a fair comparison. As such, each test case in Table 3 was run once using the SORM

\footnotetext{
${ }^{8}$ The actual time stamp is the time stamp of an aircraft obtained from the MSE.

${ }^{9}$ The earliest time stamp at arrival fix is the time stamp of arriving aircraft according to the scheduled metering fix time, as part of flight plan input.

${ }^{10}$ The unimpeded time is the time duration of an aircraft if it was the only aircraft in the airport.
} 
software with the historical runway configurations obtained from the ASPM database (i.e., the baseline), and once with the TRCM-generated runway configuration schedule. Specifically, there were a total of 72 runs (12 days $\times 3$ airports $\times 2$ runway schedule cases). The throughput metrics were computed for both baseline and TRCM cases, and compared for this assessment. For the baseline case, the software bypasses the planning cycles (the Planning in TRCM in Figure 1) and only invokes the MSE (the Execution in MSE) to simulate the airborne and ground routes of all aircraft given the historical runway configuration schedule. All simulations started with no aircraft in the airport, and ended when all aircraft had left the airport. No significant difference in run times between the baseline and TRCM cases was noticed on the same computing system. MEM had the shortest run times, and DFW had the longest run times. It is suspected that the longest run times at DFW is due to twice the traffic volumes as those at MEM and JFK. It is noticed that MEM had less runway crossing events than those at JFK. The runway crossing event causes an aircraft to leave the airport later, and, therefore, it may increase the run time. As such, even with approximately the same number of operations, MEM had shorter run times than JFK.

\section{Analysis Techniques}

To test if the baseline and TRCM runway schedules have the same average performance metrics, the well-known t-test is used [7]. However, the t-test assumption is that both schedules produce Normal-distribution metrics. Deviation from this assumed distribution requires the use of an equivalent non-parametric Mann-Whitney test [7]. The Mann-Whitney test is used to determine if the medians, as opposed to the mean, of the baseline and the TRCM metrics are the same.

\section{Tested Results}

The following section gives detailed results for all tested days. Section A provides throughput efficiency results. Although runway configuration changes are not a designated metric for this study, the frequency of changes recommended by the TRCM is evaluated for its operational feasibility and is given in Section B.

\section{A. Throughput Efficiency}

Scatter plots of TE versus demand for all hours and all days at MEM, DFW, and JFK are generated for arrivals and departures. . These plots are useful for checking sensitivity of the TE to traffic volume. Figure 3 shows the scatter plots for arrivals and departures at MEM. There is no strong trend between demand and TE. In other words, TE is insensitive (or robust) to traffic volume. Historical capacity during the period of 10/2009 to 09/2010 was retrieved from the ASPM database. Depending on many factors such as weather and runway configuration, the Airport Acceptance Rate (AAR) and Airport Departure Rate (ADR) capacities can vary from hour to hour. Therefore, they are considered as random variables with underlying distributions. The $95 \%$ coverage interval $^{11}$ of the historical capacity distribution is used to determine how close the traffic volume used in this study is to the capacity during the same time period. This interval is shown on the figure (magenta vertical lines). For arrivals, 95\% of AARs are between 56 and 100 aircraft per hour. For departures, 95\% of ADRs are at 60 aircraft per hour. It should be noted that the demand volumes used for this study contain fewer flights than what is reported in ASPM, thus making the tested demand volumes farther way from the maximum capacity.

Figures 4 to 5 show similar plots for DFW and JFK. There is no strong trend between demand and TE at these two airports. For DFW arrivals (figure 4), 95\% of AARs fall between 72 and 126 aircraft per hour. For departures, 95\% of ADRs are between 80 and 100 aircraft per hour. Both arrival and departure volumes used in this study are less than their historical capacities. For JFK arrivals and departures (figure 5), 95\% of AARs fall between 28 and 52 aircraft per hour. For departures, 95\% of ADRs are between 22 and 52 aircraft per hour. It is noted that both arrival and departure volumes in this study, which exclude military and general aviation, are at their historical capacities.

\footnotetext{
${ }^{11}$ 95\% Coverage interval indicates that 95\% of hourly throughputs fall within this interval.
} 

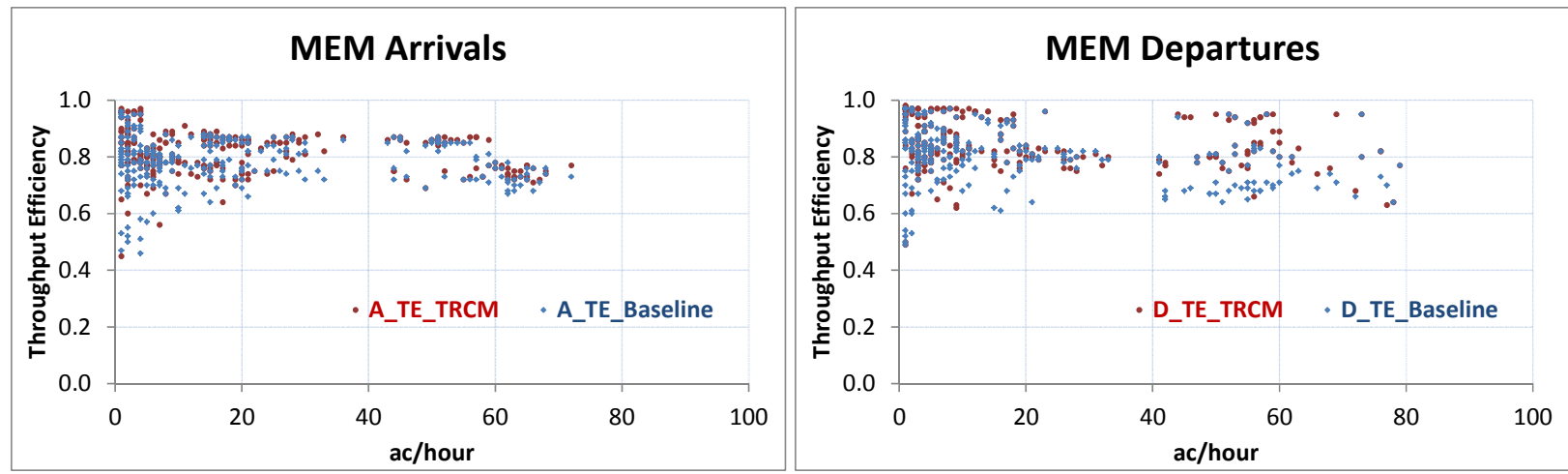

Figure 3. Scatter plots for all tested days at MEM with the $95 \%$ coverage intervals of historical Airport Acceptance Rate and Airport Departure Rate (magenta vertical lines).
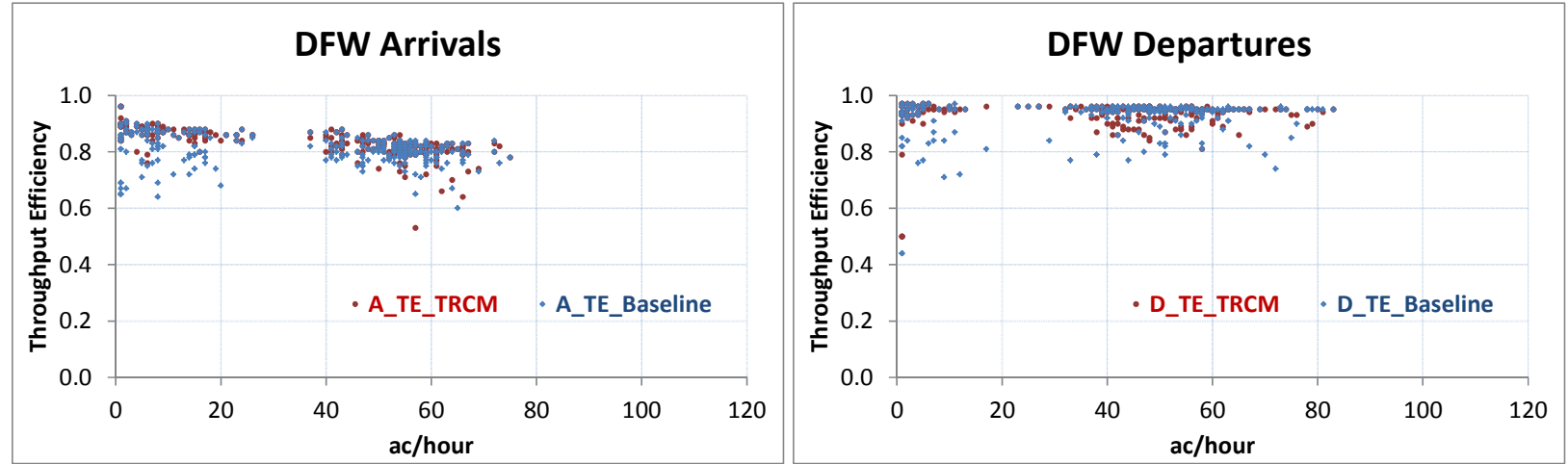

Figure 4. Scatter plots for all tested days at DFW with the $95 \%$ coverage intervals of historical Airport Acceptance Rate and Airport Departure Rate (magenta vertical lines).
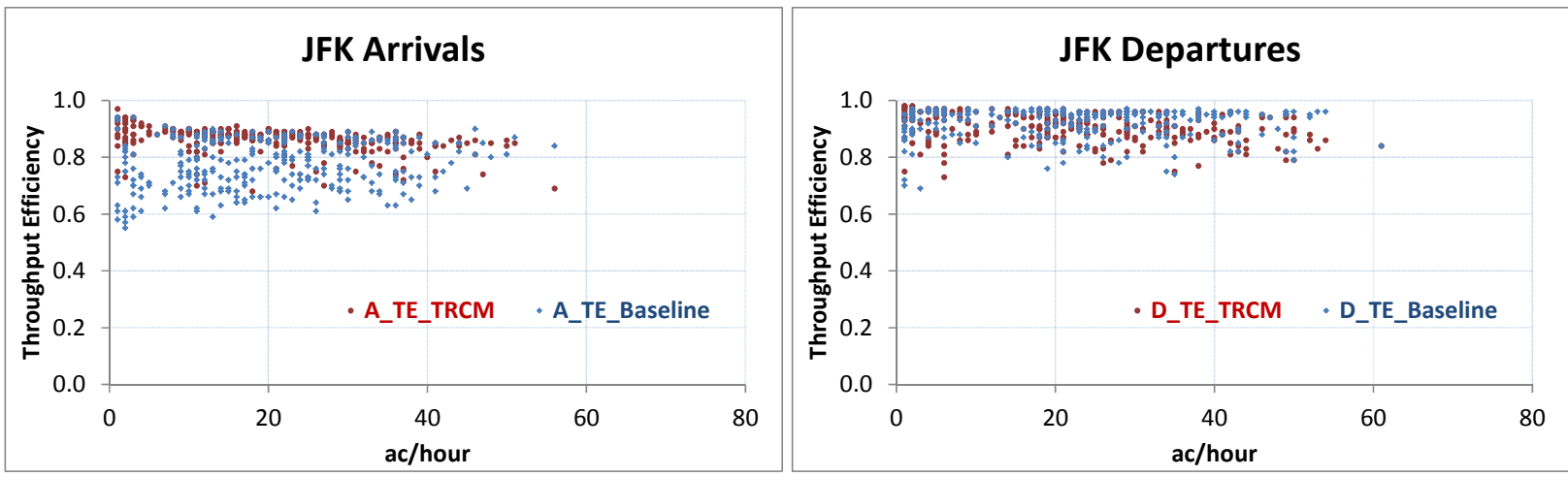

Figure 5. Scatter plots for all tested days at JFK with the $95 \%$ coverage intervals of historical Airport Acceptance Rate and Airport Departure Rate (magenta vertical lines).

Figure 6 shows mean (over all hours) TEs for arrivals and departures at all three airports. TE varies by days, type of operation (arrival vs. departure), and runway configuration schedules (baseline vs. TRCM). Table 4 summarizes the mean (over all days) and standard error by type of operation, runway configuration schedules, and airports. The standard error can be thought as uncertainty associated with the average TE. General observations can be made. The average TEs of departures are higher than arrivals for all airports; this makes sense operationally because departure schedule is more flexible (more efficient) to adjust than arrival schedule. The TE uncertainties of the TRCM are 
smaller for all airports. In other words, the baseline has more variability in their operations than the TRCM. It is intuitively suspected that the historical operations used many different criteria (or objectives) in runway configuration decision; whereas the TRCM used the same objective across all tested days. Using the TRCM schedule usually provides better TEs, in terms of averages and uncertainties, over the baseline schedule for arrivals and departures.

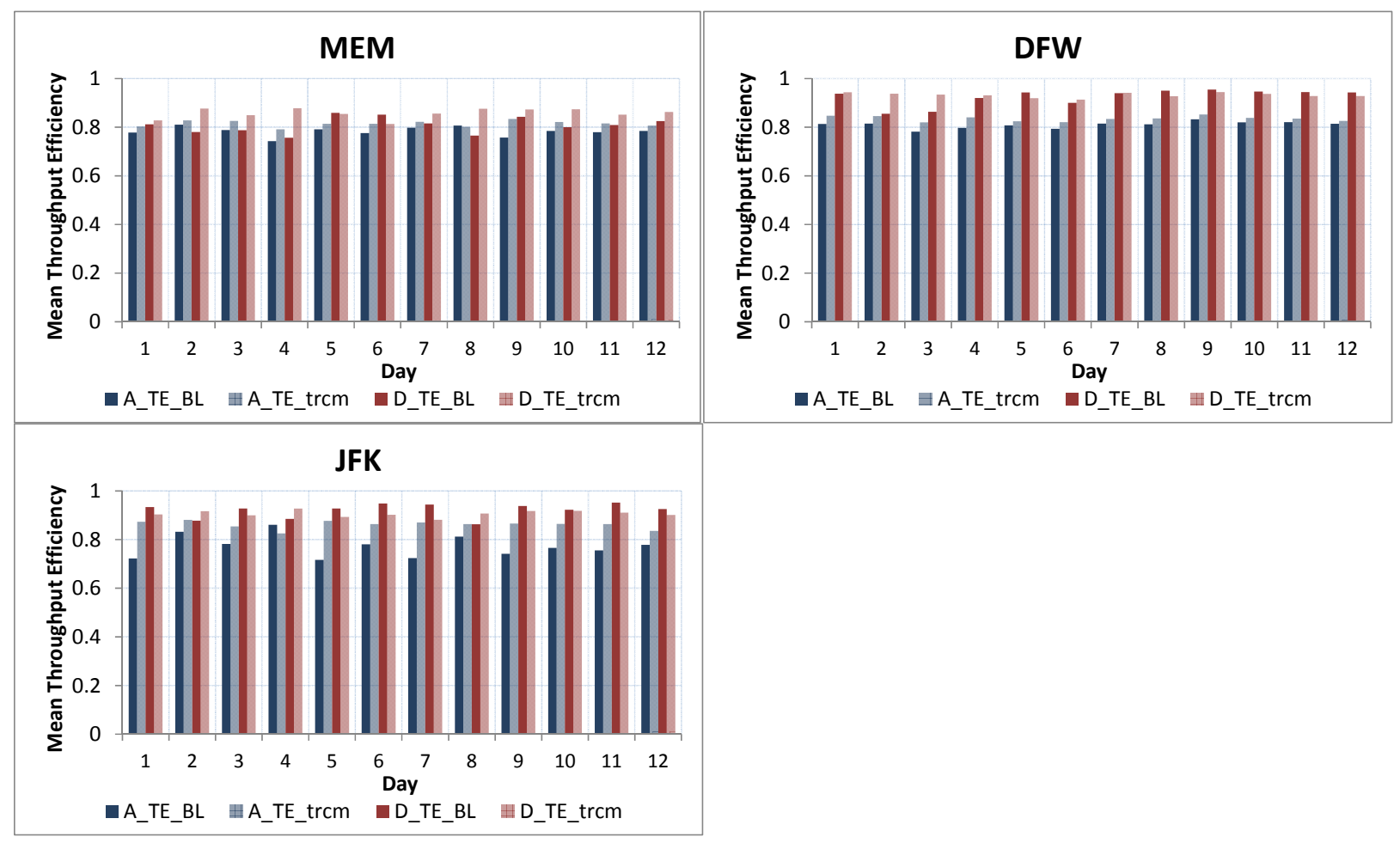

Figure 6. Mean (over all hours) throughput efficiencies (TE) at MEM, DFW, and JFK for arrivals and departures for all days.

Table 4. Summary of the average and standard error for Throughput Efficiency (TE) performances at all airports.

\begin{tabular}{|clcccc|}
\hline \multirow{2}{*}{ Airport } & Operation Type & \multicolumn{2}{c}{ Mean } & \multicolumn{2}{c|}{ Standard Error } \\
\cline { 3 - 6 } & & Baseline & TRCM & Baseline & TRCM \\
\hline \multirow{2}{*}{ MEM } & Arrivals & 0.7837 & 0.8154 & 0.0051 & 0.0045 \\
\cline { 2 - 6 } & Departures & 0.8093 & 0.8578 & 0.0061 & 0.0056 \\
\hline \multirow{2}{*}{ DFW } & Arrivals & 0.8103 & 0.8354 & 0.0033 & 0.0029 \\
\cline { 2 - 6 } & Departures & 0.9248 & 0.9324 & 0.0041 & 0.0034 \\
\hline \multirow{2}{*}{ JFK } & Arrivals & 0.7726 & 0.8618 & 0.0052 & 0.0025 \\
\cline { 2 - 6 } & Departures & 0.9208 & 0.9069 & 0.0031 & 0.0029 \\
\hline
\end{tabular}

Improvements in the mean TEs are shown in figure 7 as percent improvement with respect to the baseline. MEM provides $4 \%$ TE improvement for arrivals, and 6\% improvement for departures. At DFW and JFK, the TRCM provides higher percent improvements for arrivals (between 3 and 12 percent) than departures (between -2 and 1 percent). Especially, JFK shows a substantial TE improvement for arrivals (12\%), but a slight TE reduction for departures (-2\%). 
Mann-Whitney tests show TRCM benefits for MEM arrivals and departures (p-values $=0.0001$ ). For DFW, there is a TRCM benefit for arrivals (p-value $=0.0001$ ). For DFW departures there is no TRCM benefit. For JFK, there is a TRCM benefit for arrivals ( $\mathrm{p}$-value $=0.0001$ ), but a TRCM penalty for departures ( $\mathrm{p}$-value $=0.0001$ ).

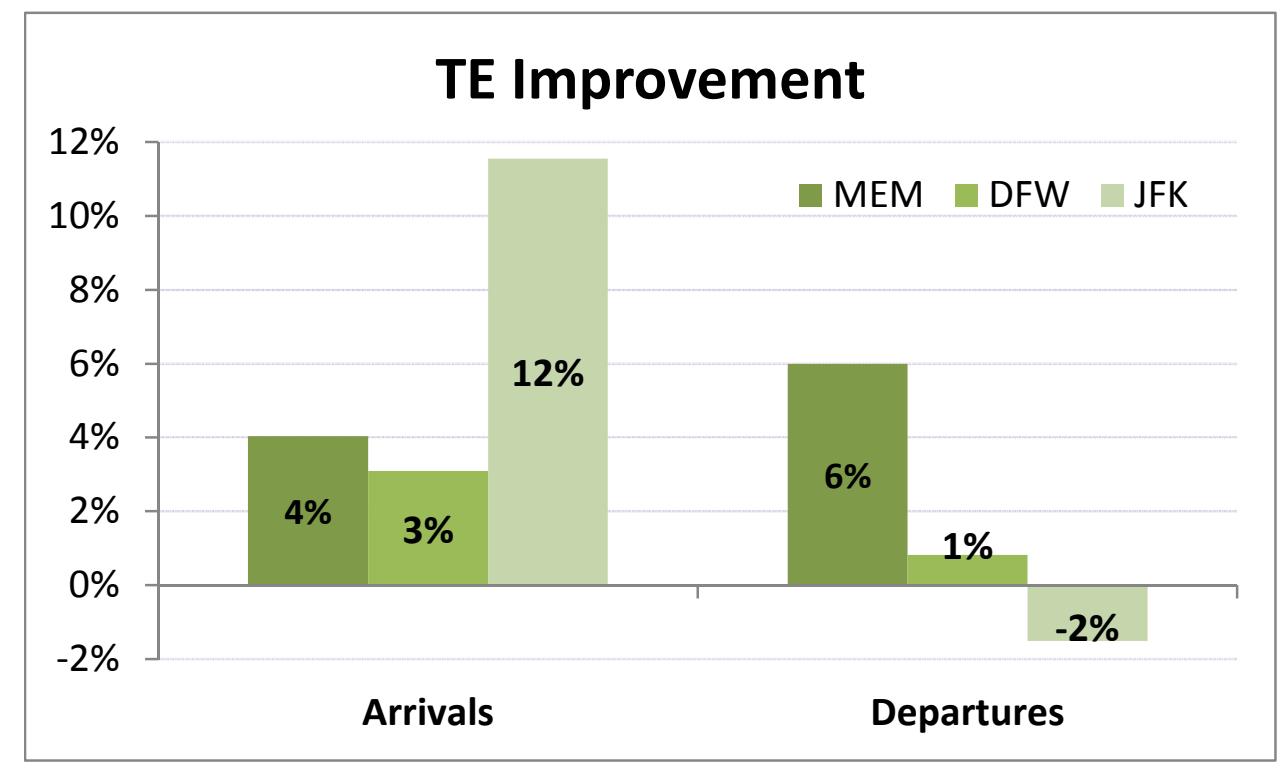

Figure 7. Average (over all days) improvement in throughput efficiency (ratio of TRCM and baseline).

\section{B. Runway Configuration Changes}

Typically, configuration changes can be categorized as: (1) a change in the primary direction of arrival and departure flows; and (2) a change in how runways are used within the same primary flow direction. Examples of the former category at JFK are a change in the southeast (13R/13L) to northwest (31L/31R) direction, or a change in the northeast (4L/4R) to southeast (13R/13L) direction. Examples of the latter category are a change in runway usage from an arrival (or departure) runway to a mixed-use runway for both arrivals and departures (i.e., from A_31R_D_31L to A_31R_31L_D_31L), or an additional runway to the existing primary flow direction (i.e., from A_13L_D_13R to A_13L_22L_D_13R).

The first category, a change in the primary flow direction, affects arrivals more than departures. Because of the way arrivals are routed into terminal areas, there must be enough airspace to allow published routes to be flown through the TRACON airspace and proper airport traffic patterns to be flown, including any additional vectoring or path-stretching that might be needed for spacing considerations. A change in the primary flow direction must account for all of these, especially for any flights that would have to be re-routed in order to accommodate the new runway configuration. Because arrivals are affected more by changes in the flow direction, the changes to flow direction that are counted for this study are those associated with a change in the primary arrival runways used. In contrast, the second category, a change in active runways within the same flow direction, requires less coordination among flights (especially for arrivals), airspace, and airport traffic pattern than the change in the flow direction.

Figure 8 shows the number of configuration changes resulting from using the TRCM schedule; changes in flow direction (the first category) are labelled as "Flow" and changes in runway usage within the same flow direction (the second category) are labelled as "Usage". The corresponding baseline number of changes that occurred on those days is also shown. The number of configuration changes varied by days, change categories ("Flow" vs. "Usage"), runway schedules (baseline vs. TRCM), and airports. Across all tested airports, the TRCM consistently has higher number of changes in flow direction than the baseline for all tested days. At MEM, the TRCM has higher number of changes in both categories than the baseline. Only at MEM, there is no usage change for the baseline for all tested days. 


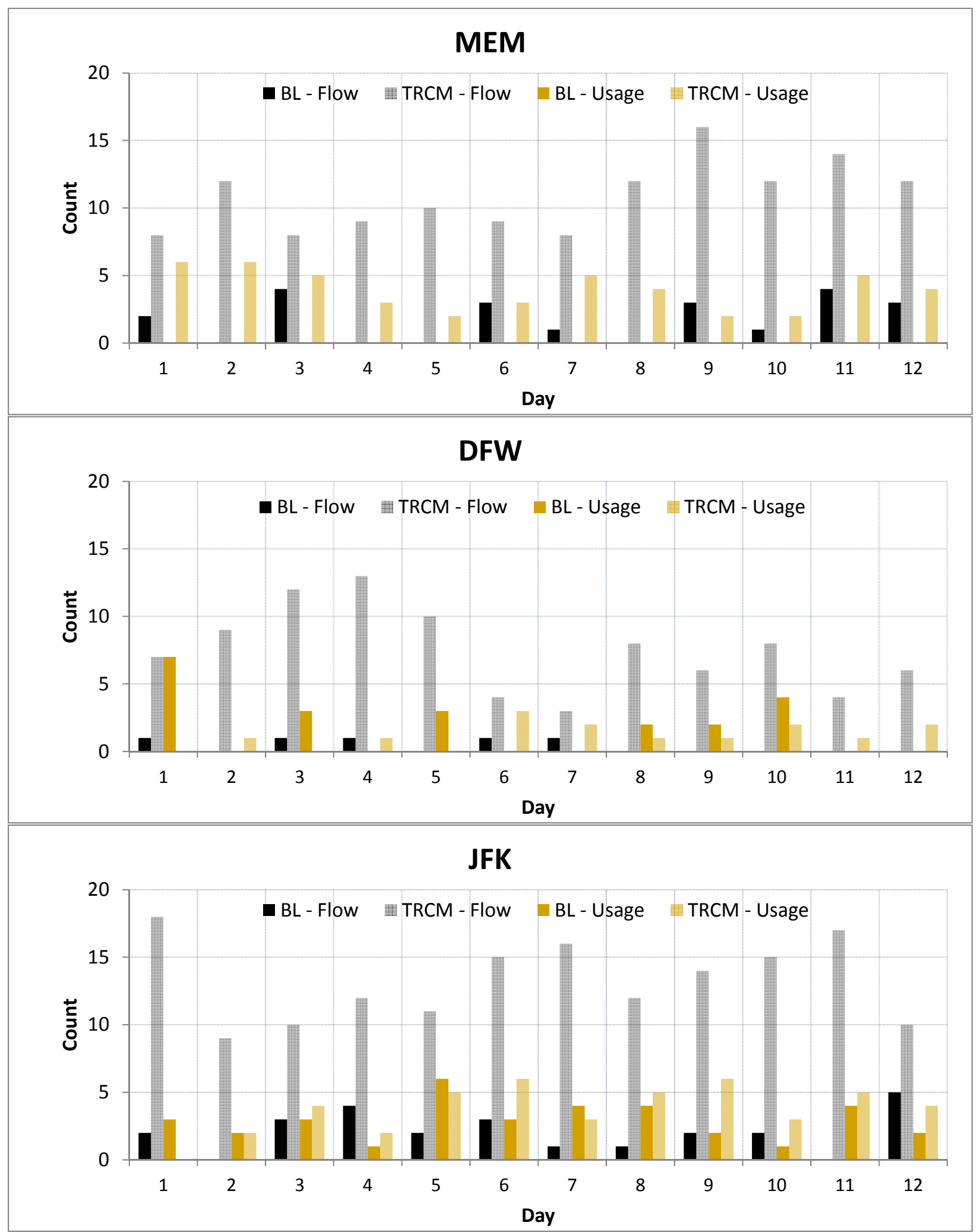

Figure 8. Number of configuration changes at MEM, DFW, and JFK for all tested days. 
Table 6 summarizes number of days, ranges (min, max), standard deviation, and mean (over all days) of the number of configuration changes by change categories, runway schedules, and airports. Historical configurations by quarters of hours during the period of 10/2009 to 09/2010 were retrieved from the ASPM database. For each day, number of historical changes was tallied by change categories. The descriptive statistics for the number of daily historical changes are also given in Table 6. The descriptive statistics of the baseline are reasonably consistent with those of the ASPM as one would expect because the baseline data is a representative sample of the historical population.

The TRCM generally results in higher mean and day-to-day variations (larger ranges and standard deviations) than the baseline and ASPM at all airports for both change categories, except for the usage change at DFW. Figure 9 plots the average change per day for all change categories, runway schedules, and airports. It is observed that the TRCM algorithm recommends more flow changes than usage changes at all airports. This implies that the algorithm finds more transit time savings in changing flow direction than in changing how runway is used. For flow direction change, the TRCM recommended about 6 times the baseline at MEM and JFK; and 18 times at DFW. These values are clearly excessive compared to what is normally found at these airports. The TRCM currently does not take into account the costs associated with a flow direction change whereas air traffic personnel are keenly well aware of the costs. Further refinement in the algorithm will need to incorporate these costs.

Table 6. Number of days (n), range (min, max), standard deviation (SD), and mean (over all days) for number of configuration changes by change categories (flow vs. usage change), runway schedules (ASPM, baseline, and TRCM), and airports.

\begin{tabular}{|c|c|c|c|c|c|c|c|c|c|c|c|c|c|}
\hline \multirow{2}{*}{$\begin{array}{c}\text { Change } \\
\text { Categor } \\
\mathbf{y}\end{array}$} & \multirow{2}{*}{$\underset{t}{\text { Airpor }}$} & \multicolumn{4}{|c|}{$\begin{array}{l}\text { ASPM Schedule } \\
(10 / 2009-09 / 2010)\end{array}$} & \multicolumn{4}{|c|}{ Baseline Schedule } & \multicolumn{4}{|c|}{ TRCM Schedule } \\
\hline & & $\mathrm{n}$ & Range & SD & Mean & $\mathrm{n}$ & Range & SD & Mean & $n$ & Range & SD & Mean \\
\hline \multirow{3}{*}{$\begin{array}{c}\text { Flow } \\
\text { Change }\end{array}$} & MEM & 364 & $0-7$ & 1.56 & 1.47 & 12 & $0-4$ & 1.60 & 1.75 & 12 & $8-16$ & 2.59 & 10.83 \\
\hline & DFW & 364 & $0-5$ & 0.77 & 0.47 & 12 & $0-1$ & 0.52 & 0.42 & 12 & 3-13 & 3.15 & 7.5 \\
\hline & JFK & 364 & $0-8$ & 1.47 & 2.27 & 12 & $0-5$ & 1.51 & 2.08 & 12 & $9-18$ & 2.99 & 13.25 \\
\hline \multirow{3}{*}{$\begin{array}{l}\text { Usage } \\
\text { Change }\end{array}$} & MEM & 364 & $0-4$ & 0.49 & 0.17 & 12 & 0 & 0 & 0 & 12 & $2-6$ & 1.51 & 3.92 \\
\hline & DFW & 364 & $0-11$ & 2.14 & 2.38 & 12 & $0-7$ & 2.22 & 1.75 & 12 & $0-3$ & 0.94 & 1.17 \\
\hline & JFK & 364 & $0-8$ & 1.65 & 2.40 & 12 & $1-6$ & 1.44 & 2.92 & 12 & $0-6$ & 1.82 & 3.75 \\
\hline
\end{tabular}

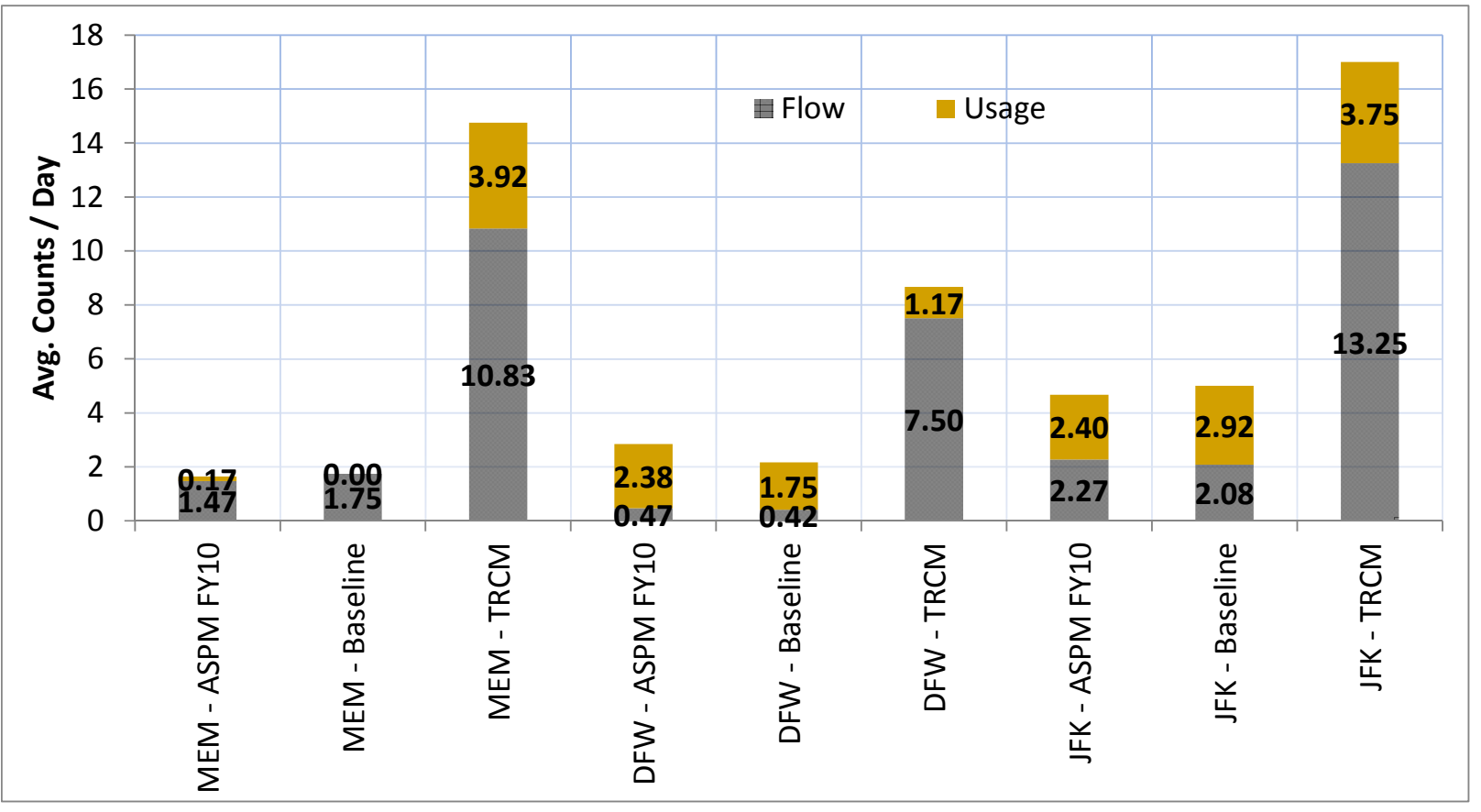

Figure 9. Average number of configuration changes per day at MEM, DFW, and JFK for all change categories and runway schedules.

12

American Institute of Aeronautics and Astronautics 


\section{Conclusion and Future Research}

The System-Oriented Runway Management (SORM) concept is a collection of needed capabilities focused on more efficient use of runways while considering all of the factors that affect runway use. Tactical Runway Configuration Management (TRCM), one of the SORM capabilities, is the process of provided runway configuration and runway usage recommendations, monitoring the active runway configuration for suitability given existing factors, based on a 90 minute planning horizon. This study provided an initial assessment of throughput benefits using twelve representative days during 2009-2010 traffic volumes at Memphis International Airport (MEM), Dallas-Fort Worth International Airport (DFW), and John F. Kennedy International Airport (JFK).

Generally, to determine that the system with TRCM provides better throughput than the system without TRCM (i.e., baseline) is to show that it can serve more flights without dramatically increasing delays. However, this study is based on historical demand, and both the baseline and TRCM have the same number of flights being served for the tested day. The analysis did not test with future projected demand or did not artificially increase traffic demand to determine whether the TRCM would be able to absorb more flights than the baseline. Nevertheless, it is valuable to determine if the throughput metric differs between the baseline and the TRCM at the representative today's volumes. Throughput metric in this study is throughput efficiency (TE). Based on the initial assessment of TE at MEM, there are statistical benefits for both arrivals and departures at MEM with an average of $4 \%$ for arrivals, and $6 \%$ for departures. For DFW, there is a statistical benefit for arrivals with an average of $3 \%$. Although there is an average of $1 \%$ benefit observed for departures, it is not statistically significant. For JFK, there is a $12 \%$ benefit for arrivals, but a $2 \%$ penalty for departures.

Across all airports, there are two consistent observations. First, the average TEs of departures are higher than arrivals; this makes sense operationally because departure schedule is more flexible (more efficient) to adjust than arrival schedule. Second, the TE uncertainties of the TRCM are smaller than those of the baseline. In other words, the baseline has more variability in their operations than the TRCM. It is intuitively suspected that the historical operations used many different criteria (or objectives) in runway configuration decision; whereas the TRCM used the same objective across all tested days. Using the TRCM schedule usually provides better TEs, in terms of averages and uncertainties, over the baseline schedule for arrivals and departures.

Although the number of runway configuration changes is not initially a metric for this study, it is evaluated to determine whether or not the number of changes recommended by the TRCM is operationally feasible. Configuration changes are categorized as: (1) a change in the primary direction of arrival flows (referred to as "Flow"); and (2) a change in how runways are used within the same primary flow direction ("Usage"). The frequency of configuration changes varied by days, change categories ("Flow" vs. "Usage"), runway schedules (baseline vs. TRCM), and airports. Overall, the TRCM recommends the higher average change per day than the baseline. This higher frequency is theoretically anticipated because the algorithm is able to take all flight, weather, and airport information into account while trading off the airborne, taxi, and queue (departing aircraft only) time durations for the minimum total time. This trade off calculation is usually complex and may be beyond operator's capability without significantly increasing the workload. The right balance between the operator's acceptable changes per day and the expected benefits needs to be further investigated so that the number of changes is not excessive.

It is observed that the TRCM algorithm recommends more "Flow" changes than "Usage" changes at all airports. This implies that the algorithm finds more transit time savings in changing flow direction than in changing how runway is used. For "Flow" change, the TRCM recommended about 6 times the baseline at MEM and JFK; and 18 times at DFW. These change frequencies are clearly excessive compared to what is normally found at the airports. As part of future algorithm improvements, it is recommended that the TRCM should

(1) limit the number of changes per day to what would be considered a more acceptable number.

(2) implement minimum benefit thresholds for a configuration change, in order for it to be worth the operational effort involved in making such a change. These thresholds could be airport-specific, or dependent on how easy to execute operationally. Thresholds could be smaller for easy-to-execute configuration change (i.e., "Usage” change) than those for hard-to-execute change (i.e., "Flow" change).

Future research should re-assess the benefits after making the suggested improvements and extend the test cases to include increased projected future demand volumes. Analyzing a significant number of additional major airports is also necessary to enable extrapolation to a NAS-wide benefits assessment. 


\section{Acknowledgments}

The authors would like to thank Steven W. Robbins at NASA Langley Research Center for his valuable assistance with the SORM software.

\section{References}

[1] Joint Planning and Development Office, 2007, "Concept of Operations for the Next Generation Air Transportation System,” 219 pp., http://www.jpdo.gov/library/nextgen_v2.0.pdf.

[2] Lohr, G., Brown, S., Stough, H., Atkins, S., Eisenhawer, S., and Long, D., “System Oriented Runway Management: A Research Update,” Ninth USA/Europe Air Traffic Management Research and Development Seminar, Berlin, Germany, June 2011.

[3] Lohr, G., Brown, S. Atkins, S. Eisenhawer, S., Bott, T., Long, D., and Hasan, S., "Progress Toward Future Runway Management," AIAA-2011-6925, Eleventh AIAA Aviation, Technology, Integration, and Operations (ATIO) Conference, Virginia Beach, VA, September 2011.

[4] Cheng, F., Gulding, J., Baszczewski, B., and Galaviz, R., “An Optimization-Based Sample Day Selection Algorithm for Future Schedule Generation,” Eleventh AIAA Aviation, Technology, Integration, and Operations (ATIO) Conference, AIAA 2011-7054, Virginia Beach, VA, September 2011.

[5] United States Government Accountability Office, April 2013, “NextGen Air Transportation System, Report to Congressional Requesters” 71 pp., GAO-13-264.

[6] Chandrasekar, S., Shin, S., and Hwang, I., “Throughput Metric Development and Analysis for the National Airspace System,” $12^{\text {th }}$ AIAA Aviation Technology, Integration, and Operations (ATIO) Conference, 2012.

[7] Montgomery, D. and Runger, G., Applied Statistics and Probability for Engineers, John Wiley \& Sons, Inc., 1994. 